\title{
Increased infiltration of macrophages to radioresistant lung cancer cells contributes to the development of the additional resistance of tumor cells to the cytotoxic effects of NK cells
}

\author{
MINGJING SHEN ${ }^{1,2^{*}}$, YONGBING CHEN ${ }^{2 *}$, LIJUN XU $^{2}$, RONGYING ZHU ${ }^{2}$, XIANG XUE $^{2}$, \\ YING TSAI ${ }^{1}$, PETER C. KENG ${ }^{1}$, SOO OK LEE ${ }^{1}$ and YUHCHYAU CHEN ${ }^{1}$ \\ ${ }^{1}$ Department of Radiation Oncology, University of Rochester School of Medicine and Dentistry, \\ Rochester, NY 14642, USA; ${ }^{2}$ Department of Cardiothoracic Surgery, \\ The Second Affiliated Hospital of Soochow University, Suzhou, Jiangsu 215004, P.R. China
}

Received November 10, 2017; Accepted April 2, 2018

DOI: 10.3892/ijo.2018.4394

\begin{abstract}
In this study, in order to investigate the effects of increased macrophage infiltration to radioresistant lung tumors in regulating natural killer (NK) cell-mediated immunity, we examined whether the treatment of radioresistant cells with conditioned medium (CM) from phorbol myristate acetate (PMA)/interleukin (IL)-4 treated THP-1 cells (used as a tumor-associated macrophage source) leads to the development of the additional resistance of tumor cells to NK cell cytotoxicity. We found that the susceptibility of THP-1 CM-treated radioresistant cells to NK cell cytotoxicity was decreased compared to the non-treated cells. In addition, it was found that such a decreased susceptibility was associated with increased programmed death receptor ligand 1 (PD-L1) and decreased natural killer group 2D (NKG2D) ligand levels in tumor cells. We further discovered that the THP-1 cells secreted a high level of IL-6, and that blocking IL- 6 action by the addition of a neutralizing antibody (Ab) for IL-6 into the THP-1 CM decreased the resistance of THP-1 CM-treated radioresistant
\end{abstract}

Correspondence to: Professor Soo Ok Lee or Professor Yuhchyau Chen, Department of Radiation Oncology, University of Rochester School of Medicine and Dentistry, 601 Elmwood Ave., Box 647, Rochester, NY 14642, USA

E-mail: soook_lee@urmc.rochester.edu

E-mail: yuhchyau_chen@urmc.rochester.edu

${ }^{*}$ Contributed equally

Abbreviations: NK, natural killer; CM, conditioned medium; PMA, phorbol myristate acetate; PD-1, programmed death receptor 1; PD-L1, programmed death receptor 1 ligand; NKG2D, NK group 2D; ULBP, UL16 binding protein; MICA, major histocompatibility complex class I chain-related molecules; LDH, lactate dehydrogenase; TAM, tumor associated macrophage

Key words: interleukin-6, macrophages, lung cancer, natural killer cell cytotoxicity, programmed death receptor 1 ligand, natural killer group 2D cells to NK cell cytotoxicity. Moreover, we discovered that MEK/Erk was the most critical IL-6 downstream signaling pathway in triggering the THP-1 CM effect; thus, the addition of MEK/Erk inhibitor to THP-1 CM enhanced the susceptibility of the THP-1 CM-treated radioresistant cells to NK cell cytotoxicity. On the whole, the findings of this study suggest the existence of a malignant loop characterized by increased macrophage infiltration into radioresistant cells which, in turn, promotes the development of the additional resistance of these cells to NK cell cytotoxicity.

\section{Introduction}

Tumor-associated macrophages (TAMs) often infiltrate into tumors and mediate their malignant progression $(1,2)$. TAMs may also exert immunosuppressive effects by impairing the proliferation/activation of T-cells (3). TAMs express inhibitory T-cell checkpoint regulators, such as programmed death receptor ligand 1/2 (PD-L1 and PD-L2) and CD80 (B7-1)/CD86 (B7-2) (4,5), which can interact with PD-1 and cytotoxic T-lymphocyte antigen-4 (CTLA-4) on T-cells, respectively, and serve to inhibit T-cell activation and subsequent tumor killing. TAMs also secrete chemoattractants to recruit cells, including myeloid-derived suppressor cells (MDSCs), immature dendritic cells (DCs) and Tregs, which further suppresses antitumor immunity (6). In addition to affecting T-cell function, the immunosuppressive roles of TAMs in affecting the natural killer (NK) cell-mediated innate immunity have also been reported. Krneta et al (7) demonsrated that NK cell activity in breast tumors was reduced upon contact with TAMs. Moreover, Crane et al (8) demonstrated that the NK group 2D (NKG2D) receptor level on NK cells was modulated by TAMs, which contributed to tumor cell evasion from NK cell cytotoxicity. On the contrary, Zhou et al (9) suggested that TAMs may help NK cells to attack colon adenocarcinoma and hepatocellular carcinoma cells. While these studies have investigated the role of TAM in affecting the receptor level on NK cells, to the best of our knowledge, no studies to date have focused on revealing the role of TAM in influencing the ligand levels on tumor cells to affect the susceptibility of tumor cells to NK cell cytotoxicity. 
In previous studies, we demonstrated that radioresistant lung cancer cells were more resistant to NK cell cytotoxicity than parental cells (10). The overexpression of PD-L1 may contribute to a poor cancer prognosis and resistance to anticancer therapeutics $(11,12)$. The downregulation of NKG2Dactivating ligands, such as UL16 binding protein 1 (ULBP1), ULBP2, ULBP3 and major histocompatibility complex class I chain-related molecules A and B (MICA and MICB) has been reported to be another mechanism responsible for tumor cells escaping from NK cell-mediated cytotoxicity (13). We have also previously demonstrated that the development of radioresistant lung cancer cells is associated with alterations in PD-L1 (upregulation) and NKG2D ligand (downregulation) levels in these radioresistant cells $(10,14)$.

In an independent previous study using phorbol myristate acetate (PMA)/interleukin (IL)-4-treated THP-1 cell as TAM source, we discovered that THP-1 cell migration to lung cancer cells was increased following radiation treatment (15). We consistently found a higher migration of THP-1 cells to radioresistant cells than to parental cells. Therefore, in the present study, we investigated whether the migrated macrophages to radioresistant cells further affects their susceptibility to the cytotoxic effects of NK cells. Radioresistant cells were treated with conditioned medium (CM) from THP-1 cells and the susceptibility of the THP-1 CM-treated cells to NK cell cytotoxicity was examined. We also examined whether the altered susceptibility of the THP-1 CM-treated radioresistant cells is mediated through the modulation of PD-L1/NKG2D ligand levels in cancer cells. Mechanistic experiments to trigger the THP-1 CM effect to alter the PD-L1/NKG2D ligand levels cancer cells were also performed in parallel.

\section{Materials and methods}

Cell culture. A549 (CLL-185 $\left.{ }^{\mathrm{TM}}\right)$ and H157 (CRL-5802 $\left.{ }^{\mathrm{TM}}\right)$ cell lines were purchased from the American Type Culture Collection (ATCC, Manassas, VA, USA) and cultured in RPMI-1640 containing 10\% FBS. We acknowledge that the H157 cell line has been demonstrated to be contaminated with the squamous cell lung carcinoma cell line, NCI-H1264 (https://web.expasy.org/ cellosaurus/CVCL_0463). However, we hereby confirm that this possible contamination is unlikely to affect the outcomes of our study. The radioresistant A549 and H157 subline cells were developed by fractionated radiation treatment for several months. Following a selection procedure as described in a previous study (10), two radioresistant subline cells, A549R26-1 and H157R24-1, were selected and used in the entire experiment. The THP-1 cell line (ATCC ${ }^{\circledast}$ TIB-202 $^{\text {TM }}$ ) was also obtained from ATCC. The cells were cultured in RPMI-1640 containing 10\% FBS and maintained in a humidified $5 \% \mathrm{CO}_{2}$ environment at $37^{\circ} \mathrm{C}$.

Isolation of primary NK cells. Peripheral blood mononuclear cells (PBMCs) were obtained from healthy donors following the approval of the Human Subject Protection Review Board of University of Rochester Medical Center (Rochester, NY, USA). The healthy volunteers provided consent on an institutional review board (IRB) approved study prior to donating their blood. Primary NK cells were purified from these PBMCs using a NK cell isolation kit (130-092-657; Miltenyi Biotec, Cambridge, MA,
USA) according to the manufacturer's instructions. Following isolation, the cells were maintained in IL-2-containing NK cell medium. The purity of the isolated cells $\left(\mathrm{CD}^{5} 6^{+} \mathrm{CD} 3^{-}\right)$ was confirmed by flow cytometric analysis following staining with anti-CD56-PE (12-0267-41; e-Bioscience, San Diego, CA, USA) and anti-CD3-Cy7 (300429; BioLegend, San Diego, CA, USA) antibodies (Abs). These primary NK cells were cultured in $\alpha$-MEM containing sodium bicarbonate (M4655; Sigma, St. Louis, MO, USA), IL-2 (100 U/ml) (200-02; Peprotech, Rocky Hills, NJ, USA), inositol (0.2 mM), 2-mercaptoethanol $(0.1 \mathrm{mM})$, folic acid $(0.02 \mathrm{mM}), 12.5 \%$ horse serum (Sigma) and $12.5 \%$ FBS (HyClone, Logan, UT, USA).

Induction of THP-1 differentiation and obtaining THP-1 CM. The THP-1 cells were differentiated by exposure to $100 \mathrm{ng} / \mathrm{ml}$ phorbol myristate acetate (PMA) for $24 \mathrm{~h}$ followed by the addition of human recombinant IL-4 (rhIL-4, $20 \mathrm{ng} / \mathrm{ml}, 200-04$; Peprotech) as previously described (16,17). THP-1 CM was obtained from the PMA/IL-4-treated THP-1 culture $48 \mathrm{~h}$ after the addition of fresh medium. For the immunofluorescence (IF) staining of the PMA- and PMA/IL-4-treated THP-1 cells, the cells were plated in a chamber slide and stained with FITC-CD206 Ab (321103; BioLegend, Dedham, MA, USA). Following the mounting procedure using mounting solution including DAPI (Ventashield, H-1200; Vector Laboratory, Burlington, ON, Canada), fluorescence images were obtained on an F-microscope (Axioskop 40, XE120-XL-0255; Zeiss, Goettingen, Germany).

Migration assays. For testing the migration of THP-1 cells to tumor cells, the THP-1 cells were placed in the upper chamber, while tumor cells (A549R26-1 and H157R24-1, $\left.5 \times 10^{4}\right)$ were placed in the lower chamber of Transwell plates (Corning, $8 \mu \mathrm{m}$ pore size, 24-well plates, no serum-containing media). Migrated macrophages at the end of $24 \mathrm{~h}$ of incubation were visualized by staining with a crystal violet (C-0775; Sigma) solution and counted under an inverted microscope (Axiovert 40; Zeiss). For experiments blocking IL-6 or chemokine (C-C motif) ligand2 (CCL)2/CCL5 actions, neutralizing Abs of IL-6 $(0.1 \mu \mathrm{g} / \mathrm{ml})$, CCL2 $(2 \mu \mathrm{g} / \mathrm{ml}$, MAB279) (both from R\&D Systems, Minneapolis, MN, USA) and/or CCL5 $(5 \mu \mathrm{g} / \mathrm{ml}$, ab9679; Abcam, Cambridge, MA) were used (species matched control IgG was used as controls). Three independent experiments (in triplicate) were carried out and quantification was obtained from average numbers of positively stained cells in 3 phases.

NKcytotoxicitytests (LDH release-based). NK cell cytotoxicity against tumor cells was analyzed using a lactate dehydrogenase (LDH) release assay as previously described $(10,14,18-22)$. The cancer cells $(2,500$ to 5,000$)$ were plated and on the following day, NK cells were added at various ratios (1:1, 1:5 and 1:15, target cells:effector cells) (all samples in triplicate). Following $4 \mathrm{~h}$ of co-culture, an aliquot of $50 \mu \mathrm{l}$ medium was used in the LDH cytotoxic assay using the LDH cytotoxic assay kit (88954; Thermo Fisher Scientific, Waltham, MA, USA). The experimental LDH release was corrected by the subtraction of the spontaneous release of effector cells at corresponding dilutions. \%Cytotoxicity $=($ experimental value - effector cell spontaneous control - target cell spontaneous control)/(target 
cell maximum control - target cell spontaneous control) x100. In examining the effects of neutralizing Abs on NK cell cytotoxicity, PD-L1 Ab $(10 \mu \mathrm{g} / \mathrm{ml}, 16-5982-82$; Invitrogen, Carlsbad, CA, USA) and U0126 (10 $\mu \mathrm{M}, 9903$; Cell Signaling Technology, Danvers, MA, USA) were used and species matched IgG and vehicle was used for control, respectively.

In vivo tumor xenograft experiments. The luciferase-tagged A549P and A549R26-1 cells $\left(1 \times 10^{6}\right)$ that were tagged by transfection with luciferase plasmid (pcDNA3.1-luc, V790-20; Addgene, Cambridge, MA, USA) using PolyFect Transfection reagent (301105; Qiagen, Toronto, ON, Canada). The luciferase-tagged cells were selected and maintained by the addition of G418 disulfate ( $400 \mu \mathrm{g} / \mathrm{ml}$; Sigma) to the culture. The cells were orthotopically injected $\left(1 \times 10^{6}\right.$ cells in media with Matrigel, 1:1 ratio in volume) into 8-week old female nude mice (NCI) (total 12 mice, average body weight $20.5 \mathrm{~g}$ ). Tumor development and volumes were monitored once a week by the In Vivo Imaging System (IVIS). The mice were divided into 2 groups ( $n=6$ per group). When luminescence reached to $5 \times 10^{5}$ to $1 \times 10^{6}$ radiance $\left(\mathrm{p} / \mathrm{sec} / \mathrm{cm}^{2} / \mathrm{sr}\right)$, mice of test group (lung site) were irradiated at 5 Gy for 5 consecutive days (23), while the remainder of the body was shielded from IR using lead blocks. Tumor size in the irradiated group and control group was monitored twice a week by IVIS for 3 weeks. At the end of the experiments, the mice were sacrificed and tumors were obtained. At the time of sacrifice, the average body weights of the mice bearing A549P-cell-derived and A549R26-1 cell-derived tumors were 28.5 and $28 \mathrm{~g}$, respectively. All animal experiments were performed under the supervision and guidelines of the University of Rochester Medical Center Animal Care and Use Committee. The facilities and programs for animal care at the University of Rochester comply with state and federal laws and NIH policies. The entire Animal Resource program is accredited by the Association for Assessment and Accreditation of Laboratory Animal Care International (AAALAC). Mice were housed in the University of Rochester Vivarium, which is in the Medical Center and supervised by the Department of Laboratory Animal Medicine. Mice were kept at a room temperature of $64-79^{\circ} \mathrm{F}$, a humidity range of $30-70 \%$, and were allowed free access to food and uncontaminated drinking water. We used university tumor burden guidelines as our humane endpoints. The mice did not experience any stress, discomfort or pain during the whole procedure of the experiment.

Histology and immunohistochemistry (IHC). The tissues obtained were fixed in $10 \%$ (v/v) formaldehyde in PBS, embedded in paraffin, and cut into $5-\mu \mathrm{m}$-thick sections. Tumor tissue sections were deparaffinized in xylene solution, rehydrated and immunostained with the IHC kit (sc2018; Santa Cruz Biotechnology, Santa Cruz, CA, USA). Tumor tissues were stained with Abs for F4/80 (1:1,000, ab6640; Abcam), IL-6 (1:1,000, MAB206; R\&D Systems, Minneapolis, MN, USA), CCL2 (1:1,000, ab9669) and CCL5 (1:1,000, ab9679; both from Abcam). After staining, tissues were counterstained with hematoxylin (H3401; Vector Laboratories). The average numbers of positively stained cells were obtained from the careful counting of 3 different randomly selected fields under a microscope (Axiostar Plus; Zeiss).
IL-6 ELISA. IL-6 levels in the supernatants of THP-1 cells, PMA-treated and PMA/IL-4-treated cells, was determined using the human IL-6 ELISA kit according to the manufacturer's instructions (550799; BD Biosciences, Franklin Lakes, NJ, USA). The secreted IL- 6 level ( $24 \mathrm{~h}$ of incubation) was normalized to the cell number.

$R N A$ extraction and reverse transcription-quantitative $P C R$ $(R T-q P C R)$. Total RNA $(1 \mu \mathrm{g})$ from the cells was subjected to reverse transcription using Superscript III transcriptase (Invitrogen). qPCR was conducted using the appropriate primers and a Bio-Rad CFX96 system (Bio-Rad Laboratories, Inc., Hercules, CA, USA) with SYBR-Green to determine the mRNA expression levels of the genes of interest. Primers used were as follows: human IL-6 forward, 5'-AAGCCAGAGCTG TGCAGATGAGTA-3' and reverse, 5'-TGTCCTGCAGCCAC TGGTTC-3'; human CCL2 forward, 5'-CAGCCAGATGC AATCAATGC-3' and reverse, 5'-GCACTGAGATCTTCCTA TTGGTGAA-3'; human CCL5 forward, 5'-GCTGTCATCC TCATTGCTACTG-3' and reverse, 5'-TGGTGTAGAAATACT CCTTGATGTG-3'; human PD-L1 forward, 5'-TATGGTGGTG CCGACTACAA-3' and reverse, 5'-TGGCTCCCAGAATTACC AAG-3'; human ULB1 forward, 5'-GCGTTCCTTCTGTGC CTC-3' and reverse, 5'-GGCCTTGAACTTCACACCAC-3'; human ULBP2 forward, 5'-CCCTGGGGAAGAAACTAAAT GTC-3' and reverse, 5'-ACTGAACTGCCAAGATCCAC TGC-3'; human ULBP3 forward, 5'-AGATGCCTGGGGA AAACAACTG-3' and reverse, 5'-GTATCCATCGGCTTCAC ACTCAC-3'; human MICA forward, 5'-ATCTTCCCTTTTG CACCTCC-3' and reverse, 5'-AACCCTGACTGCACAGA TCC-3'; human MICB forward, 5'-CTGCTGTTTCTGGCC GTC-3' and reverse, 5'-ACAGATCCATCCTGGGACAG-3'; human GAPDH forward, 5'-CTCCTCCACCTTTGACG CTG-3' and reverse, 5'-TCCTCTTGTGCTCTTGCTGG-3'. RT-PCR was performed with the following reaction conditions: $95^{\circ} \mathrm{C} 3 \mathrm{~min}$ followed by 39 cycles of, $95^{\circ} \mathrm{C}, 10 \mathrm{sec}, 55^{\circ} \mathrm{C}$, $30 \mathrm{sec}, 72^{\circ} \mathrm{C}$ for $30 \mathrm{sec}$. Quantification was analyzed by the $2^{-\Delta \Delta \mathrm{Cq}}$ method by normalized by the GAPDH level based on the study by Livak and Schmittgen (24). For inhibitor experiments, Janus kinase (JAK) inhibitor 1 (5 $\mu \mathrm{M}$; CAS457081-03-7; Calbiochem, San Diego, CA, USA), AG490 (50 $\mu$ M, T3434; Sigma), Stattic (10 $\mu \mathrm{M}$; CAS19983-44-9; Calbiochem), LY294002 (5 $\mu \mathrm{M}$; 440202; Sigma), U0126 (10 $\mu \mathrm{M}$; 9903; Cell Signaling Technology), SB203580 (10 $\mu \mathrm{M}$; 559387), Bay11-7082 (5 $\mu \mathrm{M}$; B5556), rapamycin (100 nM; R8781) (all from Sigma) that inhibit the JAK, JAK/signal transducer and activator of transcription 3 (Stat3), Stat3, PI3K/Akt, MEK/extracellular-signal-regulated kinase (Erk), mitogenactivated protein kinase (MAPK), nuclear factor (NF)- $\kappa \mathrm{B}$ and mammalian target of rapamycin (mTOR) pathways, respectively, were added to the radioresistant lung tumor cells.

Western blot analysis. The cells were lysed in RIPA buffer (50 mM Tris- $\mathrm{Cl}$ at $\mathrm{pH} 7.5,150 \mathrm{mM} \mathrm{NaCl}, 1 \% \mathrm{NP}-40,0.5 \%$ sodium deoxycholate, $1 \mathrm{mM}$ EDTA, $1 \mu \mathrm{g} / \mathrm{ml}$ leupeptin, $1 \mu \mathrm{g} / \mathrm{ml}$ aprotinin and $0.2 \mathrm{mM}$ PMSF). After protein determination by BCA method, proteins (20-40 $\mu \mathrm{g})$ were separated on a $8-10 \%$ SDS/PAGE gel and then transferred onto PVDF membranes (Millipore, Billerica, MA, USA). Following the blocking procedure (blocking using 5\% milk in PBS, 30 min 


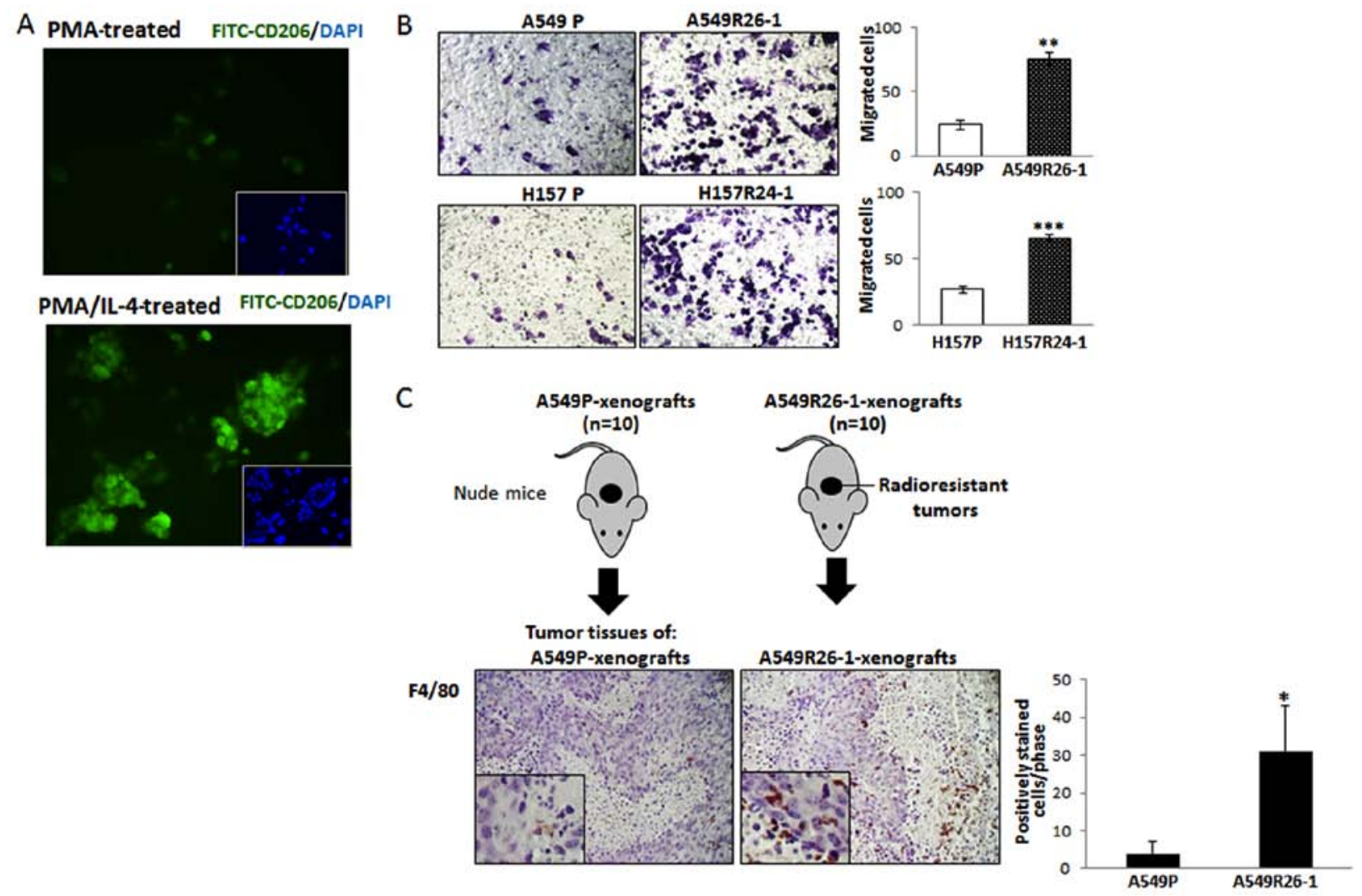

Figure 1. Increased macrophage migration into radioresistant lung cancer cells compared to parental cells. (A) CD206 immunofluorescence (IF) staining of PMA- and PMA/IL-4-treated THP-1 cells. Cells were stained with FITC-CD206 and an image was taken under an F-microscope. DAPI staining is shown in the inlets. (B) THP-1 migration to parental vs. radioresistant A549 and H157 lung cancer cells. Migration of the PMA/IL-4-treated THP-1 cells (in upper chamber) to either parental (A549P, H157P) or radioresistant A549 and H157 (A549R26-1, H157R24-1) cells (in lower chamber of Transwell plates) after $24 \mathrm{~h}$ of incubation was investigated. Membranes were stained with crystal violet solution and the average numbers of migrated THP-1 cells in randomly selected 3 areas counted under a microscope are shown. Magnification, x20. (C) F4/80 immunohistochemical (IHC) staining of tumor tissues of A549P cells-derived and A549R26-1 cells-derived xenografts. Error bars and significance values were obtained by counting positively stained cells in one randomly chosen phase of slides of 3 different stains. Magnification, $\mathrm{x} 20$ (inlet magnification, $\mathrm{x} 100$ ). ${ }^{*} \mathrm{P}<0.05,{ }^{* *} \mathrm{P}<0.01,{ }^{* * *} \mathrm{P}<0.001$.

at room temperature), membranes were incubated with primary Abs $(1: 1,000)$ and HRP-conjugated secondary Abs (goat anti-rabbit-HRP, 170-6515; goat anti-mouse-HRP, 170-6516) $(1: 5,000)$, and visualized in Imager (both from Bio-Rad Laboratories, Inc.) using the enhanced chemiluminescent (ECL) solution (34095; Thermo Fisher Scientific). The Abs used were PD-L1 (MAB1086; R\&D Systems), p-Erk (4695; Cell Signaling Technology), Erk (ab54230), IL-6 (ab6672) (both from Abcam) and GAPDH (2118S; Cell Signaling Technology). For quantification of the bands, the software of the Chemi Doc ${ }^{\mathrm{TM}} \mathrm{XRS}^{+}$ imaging system (Bio-Rad Laboratories, Inc.) was used.

Statistical analysis. The data are presented as the means \pm SEM. Differences in mean values between 2 groups were analyzed by a two-tailed Student's t-test. In comparing multiple groups, we used ANOVA with a post hoc (Tukey-Kramer) test. A value of $\mathrm{P} \leq 0.05$ was considered to indicate a statistically significant difference.

\section{Results}

Higher numbers of THP-1 cells migrate to radioresistant lung cancer cells than to parental cells. We developed radioresistant A549 (A549R26) and H157 (H157R24) cells by treating parental A549 (A549P) and H157 (H157P) cells with fractionated radiation treatment (total 26 and 24 Gy treatment for A549 cells and H157 cells, respectively). From plating these cells at a low density, several colonies were selected and expanded and the subline cells that exhibited the most significant radioresistance (A549R26-1 and H157R24-1) were selected and used in further experiments. The in vitro and in vivo results confirming the radioresistance of these cells were shown our previous study (10).

THP-1 cells are a human monocyte cell line often used for macrophage differentiation. As M2 macrophages (TAM) are activated by IL-4 produced by $\mathrm{CD} 4^{+} \mathrm{T}$-cells, PMA/IL-4-treated THP-1 cells are often used as a TAM source $(17,25)$. Following the differentiation induction process of THP-1 cells by PMA, IL-4 was subsequently added. As previously demonstrated by Xu et al (25), we found that the PMA/IL-4-treated THP-1 cells became larger and adherent, and were positively stained with the M2 type macrophage marker, CD206 (26,27) (Fig. 1A). These PMA/IL-4-treated THP-1 cells were used as the TAM source in the entire experiment. We first examined the migration of these PMA/IL-4-treated THP-1 cells to A549P vs. A549R26-1 and $\mathrm{H} 157 \mathrm{P}$ vs. H157R24-1 cells. In migration assays, we found that the A549R26-1 and H157R24-1 cells recruited more THP-1 cells than the parental cells (Fig. 1B).

Higher numbers of macrophages migrate into radioresistant lung tumors than radiation-sensitive tumors in vivo. In our 

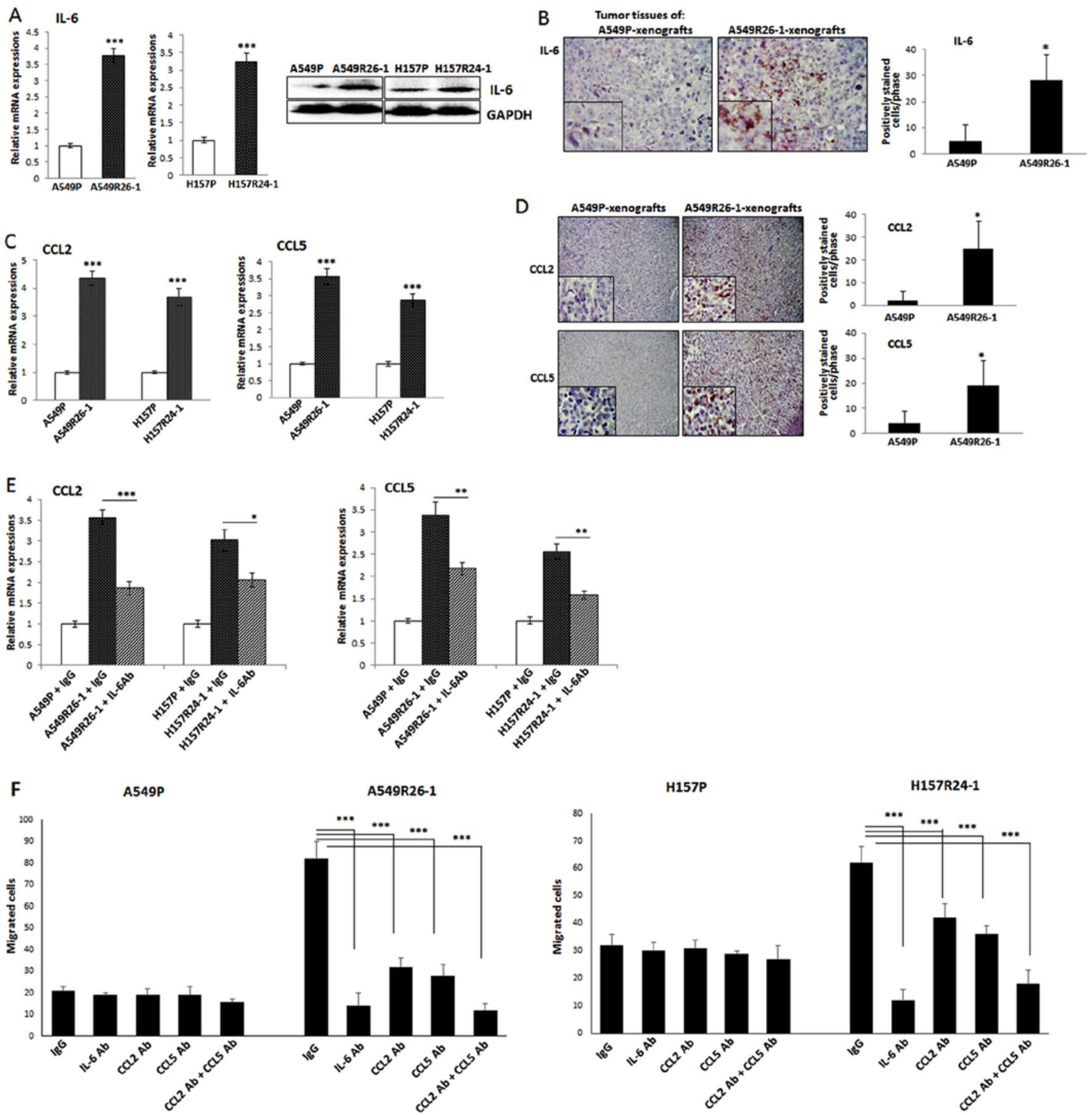

Figure 2. Examining the role of IL-6-CCL2/CCL5 in recruiting macrophages to radioresistant lung cancer cells. (A) IL-6 levels in parental vs. radioresistant lung cancer cells. (B) IL-6 level in tumor tissues obtained from A549P-xenografts and A549R26-1 xenografts. Error bars and significance values were obtained by counting positively stained cells in one randomly chosen phase of slides of 3 different stains. Magnification, x20 (inlet, magnification, x100) (C) CCL2 and CCL5 levels in parental vs. radioresistant lung cancer cells. (D) IHC staining of CCL2/CCL5 in tumor tissues obtained from A549P-xenografts and A549R26-1 xenografts. Error bars and significance values were obtained by counting positively stained cells in one randomly chosen phase of slides of 3 different stains. Magnification, x20 (inlet magnification, x100). (E) CCL2 and CCL5 levels in radioresistant lung cancer cells after treating cells with neutralizing $\mathrm{Ab}$ of IL-6 (species matched IgG as control). (F) Effects of blocking the IL-6 or CCL2 and/or CCL5 action on attracting macrophages to radioresistant lung cancer cells. A macrophage migration assat was performed after adding neutralizing antibodies to IL-6 (0.1 $\mu \mathrm{g} / \mathrm{ml})$ or CCL2 (2 $\mu \mathrm{g} / \mathrm{ml})$ and/or CCL5 $(5 \mu \mathrm{g} / \mathrm{ml})$ (species matched IgG as control) into lower chamber of Transwell plates containing radioresistant lung cancer cells. Quantification results are shown. ${ }^{*} \mathrm{P}<0.05,{ }^{* *} \mathrm{P}<0.01$ and ${ }^{* * *} \mathrm{P}<0.001$.

previous study, we developed orthotopic xenografts in nude mice by injecting A549R26-1 and A549P cells and confirmed the radioresistance of tumors derived from A549R26-1 cells compared to those derived from A549P cells [radioresistance was evaluated by tumor regression difference upon radiation treatment, data shown in our previous study (10)]. We examined macrophage infiltration to the tumor tissues of A549R26-1-xenografts and A549P-xenografts by IHC staining. We detected higher numbers of mouse macrophage marker F4/80-positively stained cells in the tumors derived 
A
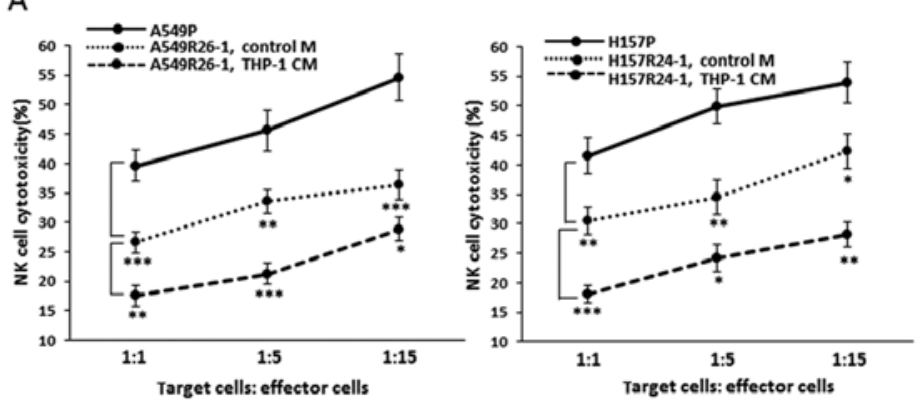

C
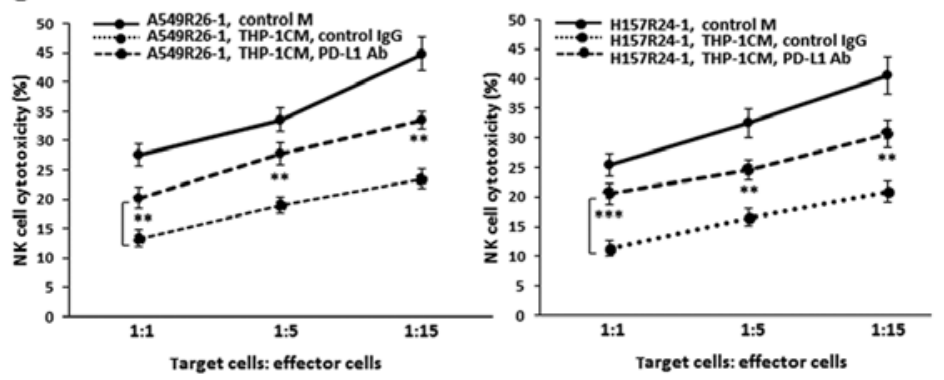

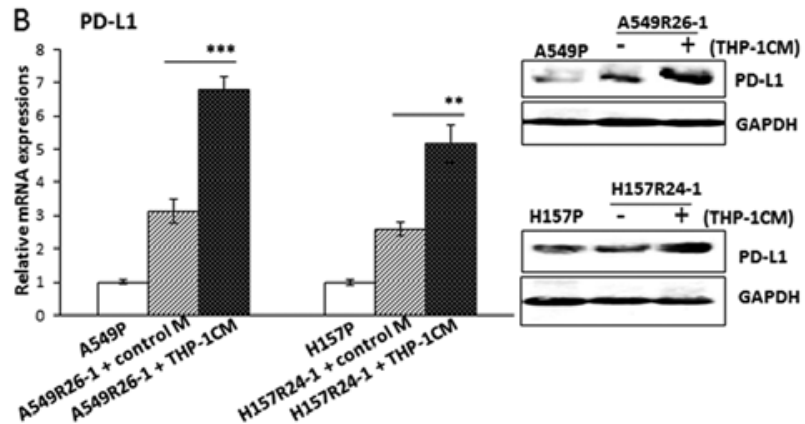

D

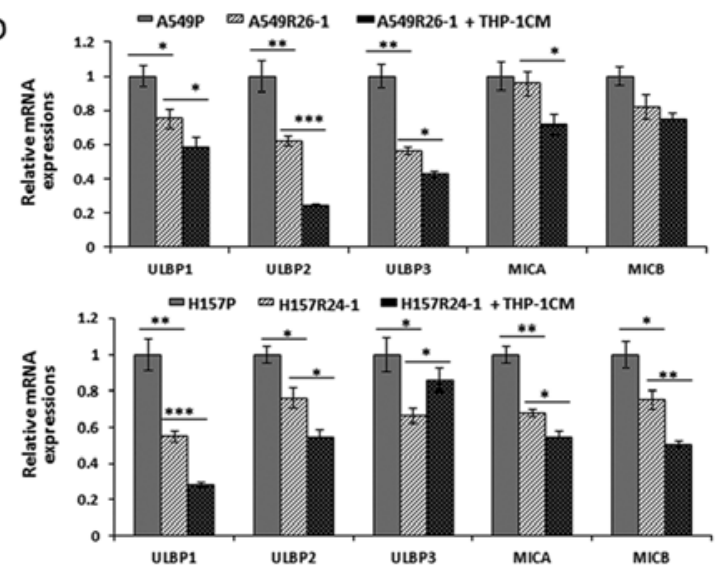

Figure 3. Examining the susceptibility of radioresistant lung cancer cells to natural killer (NK) cell cytotoxicity after treating the cells with conditioned medium (CM) of PMA/IL-4-treated THP-1 cells. (A) LDH release-based NK cell cytotoxicity tests to parental cells, radioresistant A549/H157 cells, with or without THP-1 CM treatment (12 h of incubation, control medium was used as control). Left panel shows data with the A549 cell set and right panel shows data with the H157 cell set. (B) PD-L1 levels in radioresistant A549 and H157 cells following incubation with CM of PMA/IL-4-treated THP-1 cells or control medium (left panel, qPCR results; right panel, western blot analysis results). (C) Enhanced susceptibility of the THP-1 CM-treated radioresistant lung cancer cells when PD-L1 antibody was added into tumor cell/NK cell co-culture. (D) NKG2D ligand levels (mRNA) in radioresistant A549 and H157 cells following incubation with CM of PMA/IL-4-treated THP-1 cells or control medium. ${ }^{*} \mathrm{P}<0.05,{ }^{* *} \mathrm{P}<0.01$ and ${ }^{* * *} \mathrm{P}<0.001$.

from A549R26-1 cells than in tumors derived from A549P cells (Fig. 1C), suggesting that higher numbers of macrophages infiltrated into radioresistant tumors than radiosensitive tumors.

IL-6-CCL2/CCL5 signaling may be important in recruiting high numbers of THP-1 cells to radioresistant lung cancer cells. In a previously published in vitro and in vivo study, we demonstrated that IL-6 signaling was elevated in parental lung cancer cells following radiation treatment compared to non-irradiated cells and such elevated IL-6 signaling was important in attracting macrophages to lung tumors (15). Consistent with this result, in this study, we detected higher IL-6 levels in the A549R26-1 and H157R24-1 cells than in the respective parental cells (Fig. 2A, left panel, mRNA level; right panel, protein level), and in tumor tissues derived from A549R26-1 cells than in those derived from A549P cells (Fig. 2B).

In a previous study, we revealed CCL2 and CCL5 molecules as the most critical IL- 6 downstream chemokines that are important in macrophage infiltration to tumors (15). Thus, in this study, we examined whether CCL2 and CCL5 expression was also higher in radioresistant cells than in parental cells. We observed higher levels of CCL2 and CCL5 in radioresistant cells (Fig. 2C) than in parental cells, and consistently detected higher numbers of CCL2/CCL5-positively stained cells in the radioresistant tumors than in the radiation-sensitive tumors (Fig. 2D). To confirm the IL-6 regulation of CCL2/CCL5 molecules, we added a neutralizing Ab to IL-6 and examined whether CCL2/CCL5 levels decreased. We observed a decreased CCL2/CCL5 expression in the radioresistant cells when IL-6 Ab was added to the culture (Fig. 2E), confirming the IL-6 regulation of CCL2/CCL5 molecules in radioresistant cells. We then examined whether macrophage infiltration to radioresistant cells decreases when neutralizing Abs to IL-6, CCL2 and/or CCL5 were added to the culture. We observed that the migration of the THP-1 cells to radioresistant cells was inhibited when Abs to IL-6, CCL2 and/or CCL5 were added to the lower chamber of Transwell plates containing tumor cells; however, the THP-1 cell migration to parental cells was not affected (Fig. 2F), confirming the IL-6-CCL2/CCL5 signaling role in recruiting the macrophages into radioresistant cells.

Decreased susceptibility of radioresistant lung cancer cells to NK cell cytotoxicity following treatment with CM from THP-1 cells (THP-1 CM). We then examined whether the susceptibility of radioresistant lung cancer cells to NK cell cytotoxicity could be further decreased upon treatment with THP-1 CM. We obtained THP-1 CM from the PMA/IL-4-treated THP-1 culture $48 \mathrm{~h}$ after the addition of fresh media. To monitor NK cell cytotoxicity to tumor cells, the LDH release-based NK cytotoxicity test $(10,14,18-22)$ was applied. For the NK cell source, primary NK cells that were isolated from the peripheral blood mononuclear cells (PBMCs) of healthy donors via 
A

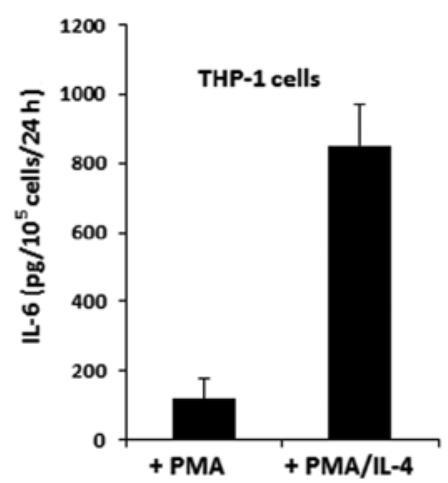

B

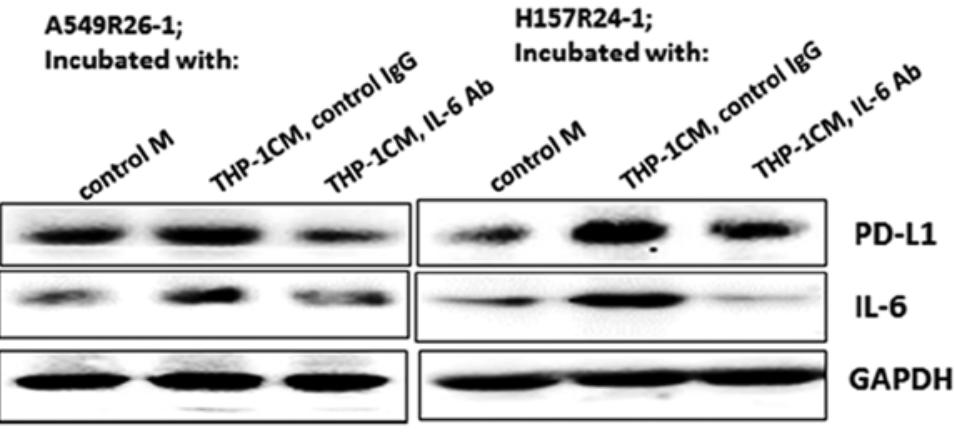

C

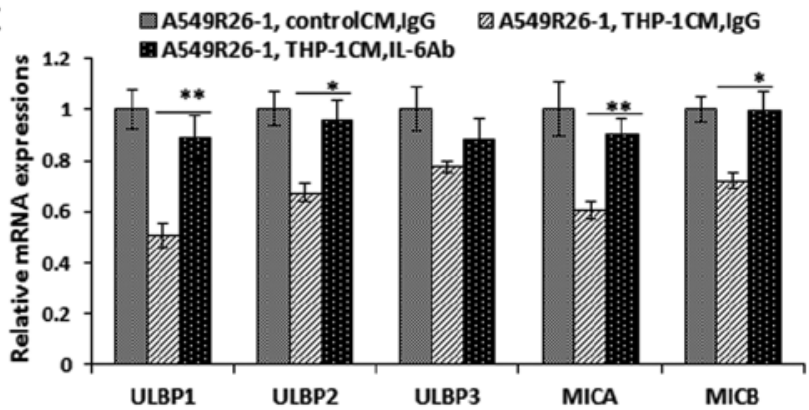

D
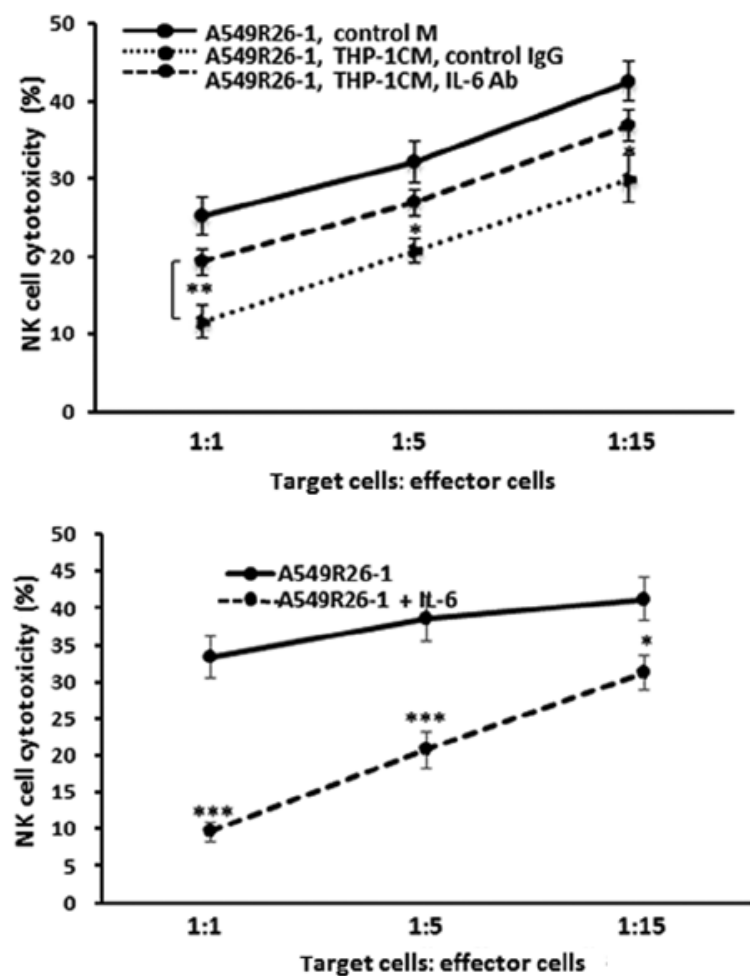
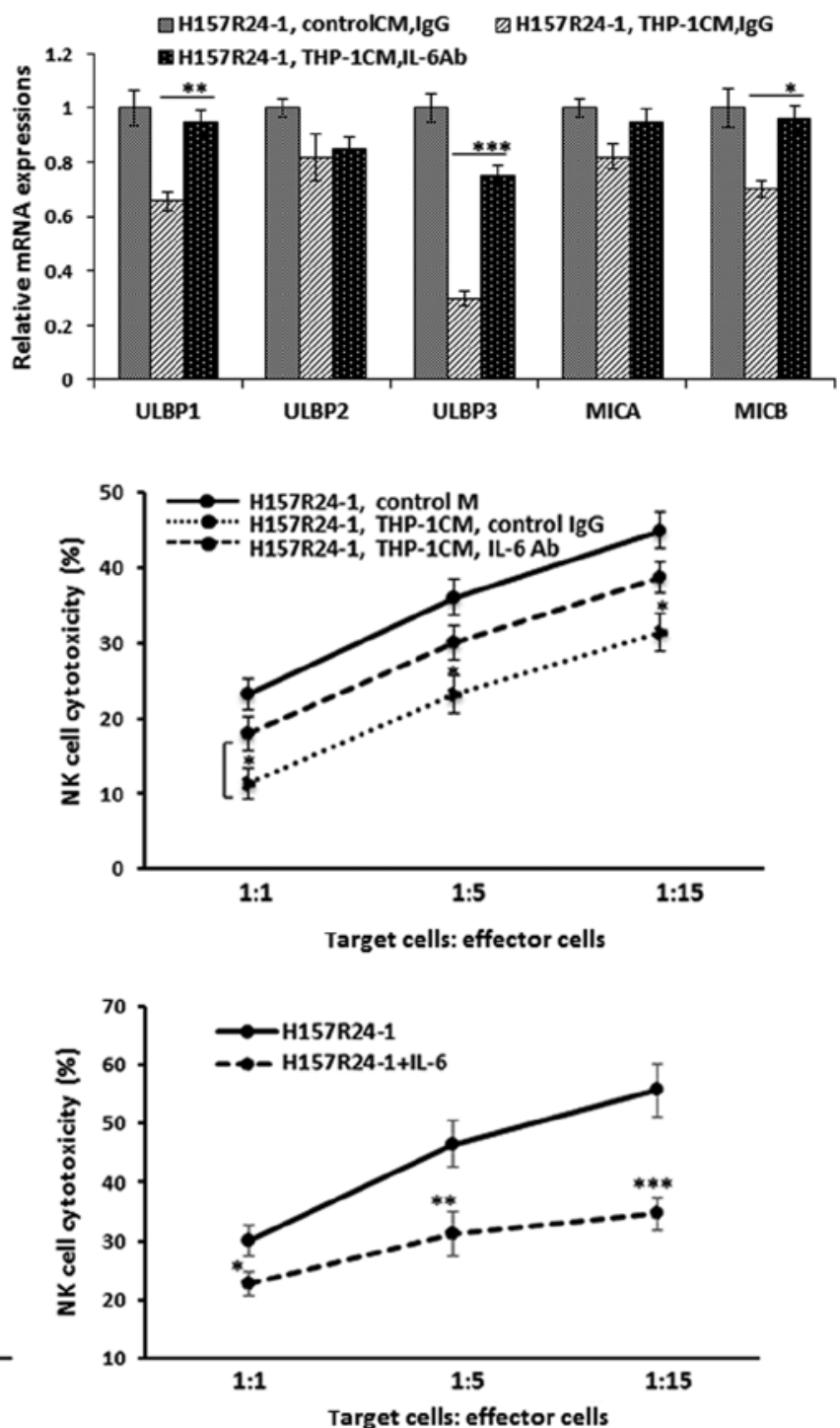

Figure 4. Examining the effects of the presence of IL-6 in conditioned medium (CM) of PMA/IL-4-treated THP-1 cells on altering the PD-L1/NKG2D ligand levels. (A) IL-6 levels (left panel, mRNA level; right panel, secreted IL-6 level) in THP-1 cells, PMA-treated and PMA/IL-4-treated. For measuring the secreted IL-6 level, IL-6 ELISA was performed. (B) Examining the THP-1 CM effects on altering the PD-L1 level in radioresistant lung cancer cells after the addition of neutralizing antibody (Ab) to IL-6 $(0.1 \mu \mathrm{g} / \mathrm{ml})$ (left panel, qPCR analysis data; right panel, western blot analysis data). (C) Examining the effect of CM of PMA/IL-4-treated THP-1 cells on altering NKG2D ligand levels (mRNA) in radioresistant lung cancer cells after the addition of neutralizing Ab of IL-6. IL-6 Ab $(0.1 \mu \mathrm{g} / \mathrm{ml})$ was added to THP-1 CM when radioresistant A549 and H157 cells were treated with THP-1 CM. (D) Natural killer (NK) cell cytotoxicity assay of radioresistant lung cancer cells treated with CM of PMA/IL-4-treated THP-1 cells after the addition of IL-6 Ab (0.1 $\mu \mathrm{g} / \mathrm{ml})$ (or control $\mathrm{IgG}$ ) into the tumor cell/NK cell co-culture. (E) NK cell cytotoxicity assay of radioresistant lung cancer cells upon the addition of rhIL-6 (50 ng/ml) into the tumor cell/NK cell co-culture. ${ }^{*} \mathrm{P}<0.05,{ }^{* *} \mathrm{P}<0.01$ and ${ }^{* * *} \mathrm{P}<0.001$.

the magnetic beads isolation method were used in all the experiments. Following the isolation of primary NK cells, the purity (>90\%) of $\mathrm{CD}^{-} 6^{+} \mathrm{CD} 3^{-} \mathrm{NK}$ cells was confirmed by flow cytometric analyses as shown in a previous study (28). 


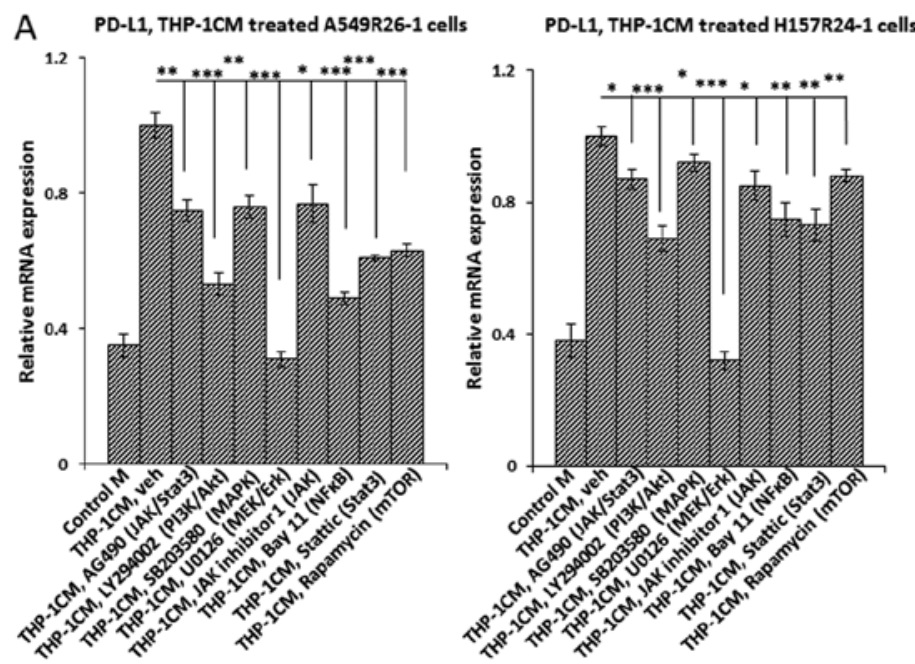

B

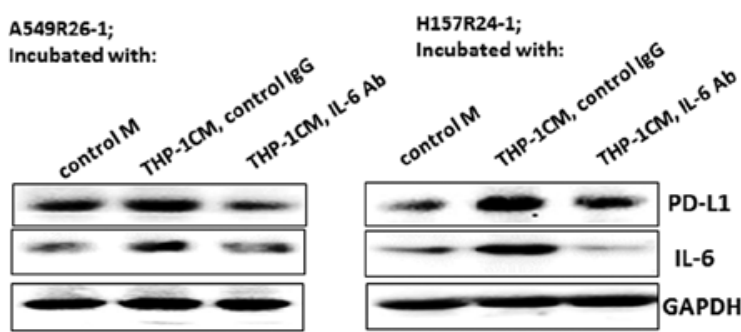

C
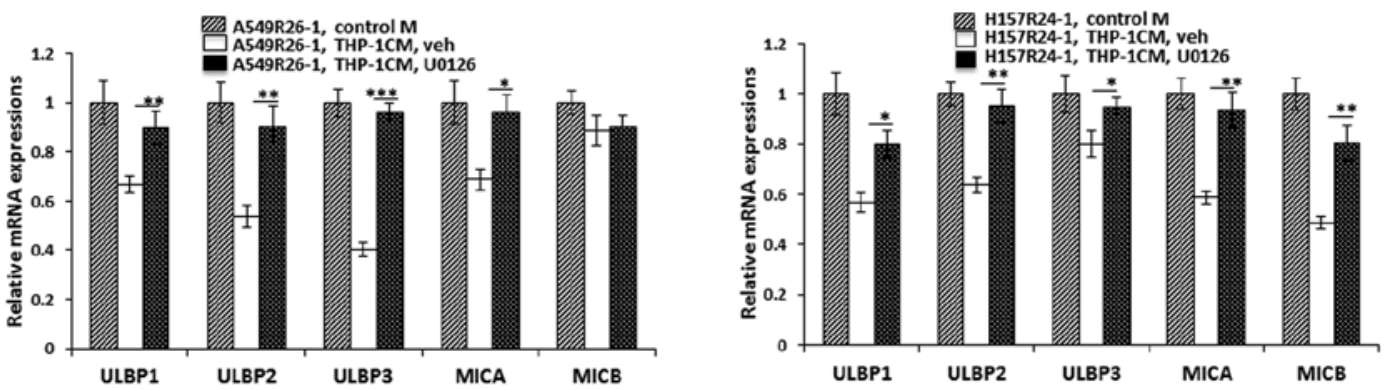

D
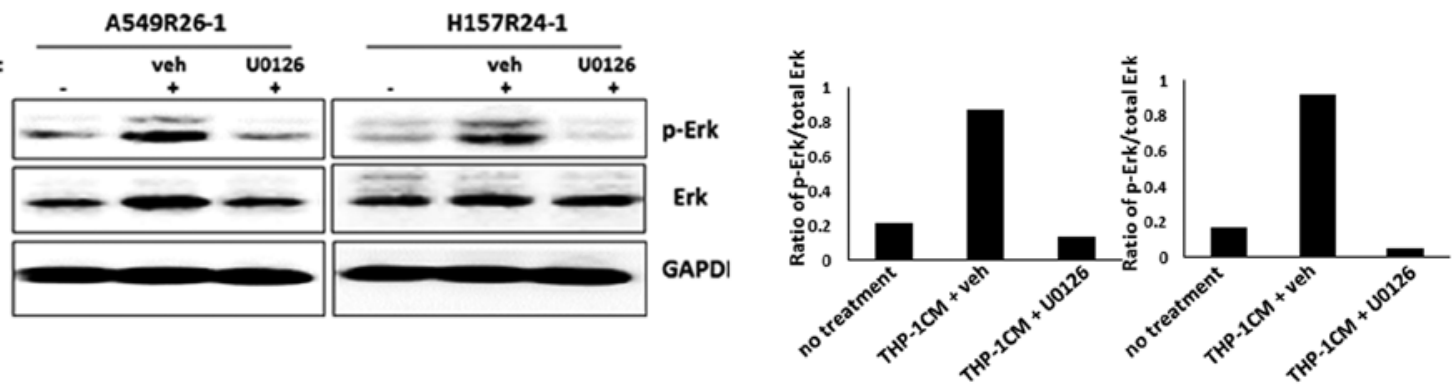

Figure 5. Searching for the IL-6 downstream signaling that is responsible for triggering the THP-1 CM effect. (A) RT-qPCR analyses of PD-L1 in radioresistant lung cancer cells treated with CM of PMA/IL-4-treated THP-1 cells after the addition of inhibitors of individual candidate signaling pathways. (B) Western blot analysis of the PD-L1 level in radioresistant lung cancer cells treated with conditioned medium (CM) of PMA/IL-4-treated THP-1 cells, with the addition of the MEK/Erk inhibitor, U0126. (C) RT-qPCR analysis of NKG2D ligand levels in radioresistant lung cancer cells treated with CM of PMA/IL-4-treated THP-1 cells after the addition of the MEK/Erk inhibitor, U0126. Left panel shows data of A549R26-1 cells and right panel shows data of H157R24-1 cells. (D) Western blot analyses of p-Erk and total Erk levels in radioresistant lung cancer cells treated with CM of PMA/IL-4-treated THP-1 cells, with the addition of U0126. Quantification of p-Erk to total Erk and GAPDH is shown in the lower panels. ${ }^{*} \mathrm{P}<0.05,{ }^{* *} \mathrm{P}<0.01$ and ${ }^{* * * *} \mathrm{P}<0.001$.

In NK cell cytotoxicity tests, consistent with our previous study (10), we observed decreased susceptibilities of radioresistant lung cancer cells to NK cell cytotoxicity compared with the parental cells (Fig. 3A). Furthermore, we observed that radioresistant cells exhibited a significantly higher resistance to NK cell cytotoxicity following treatment with THP-1 CM than the control medium-treated cells (Fig. 3A, left panel, the data with A549 cell set; right panel, with H157 cell set), suggesting that the infiltration of macrophages to radioresistant lung cancer cells may promote the development of the additional resistance radioresistance of lung cancer cells to NK cell cytotoxicity.

THP-1 CM treatment triggers the upregulation of PD-L1 and downregulation of NKG2D ligands in radioresistant lung cancer cells. The importance of the PD-L1/PD-1 immune checkpoint in regulating the NK cell-mediated immune function to cancer cells has been demonstrated in previous studies (29), as well as in our previous studies $(10,14,22)$. Thus, in this study, we examined whether the treatment of radioresistant lung cancer cells with THP-1 CM alters the PD-L1 level in tumor cells. As shown in Fig. 3B, THP-1 CM treatment triggered the upregulation of PD-L1 in radioresistant lung cancer cells, suggesting that this modulation may be associated with the THP-1CM effect we observed. When PD-L1 Ab was added to the tumor cell/NK cell co-culture, a decreased susceptibility of THP-1 CM-treated radioresistant lung cancer cells to NK cell cytotoxicity was observed (Fig. 3C), suggesting that the upregulation of PD-L1 is important in developing the additional resistance of THP-1 CM-treated radioresistant cells to NK cell cytotoxicity.

The NKG2D ligand/NKG2D interaction has been reported as important in activating the NK cell-mediated immune func- 

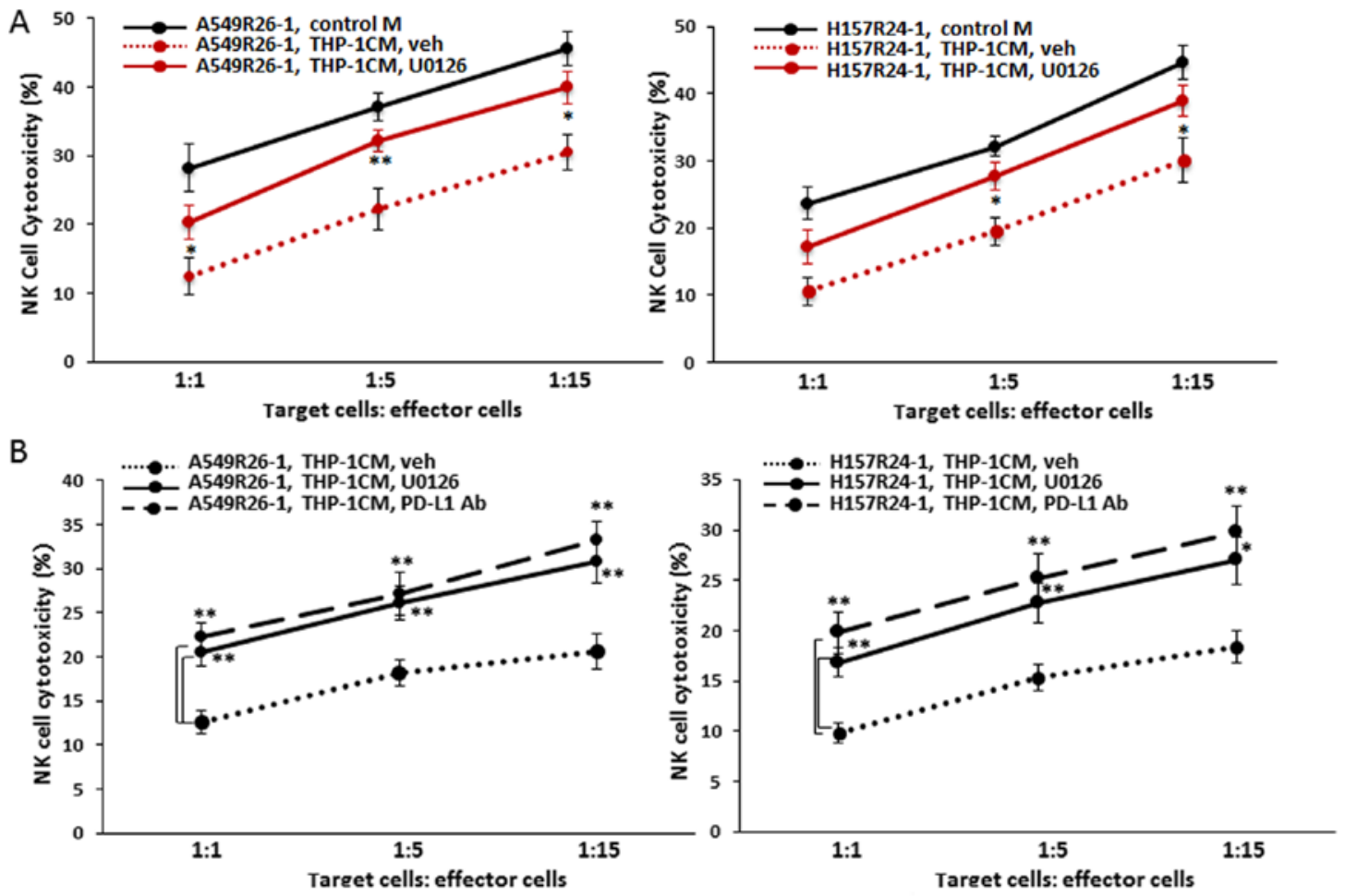

C

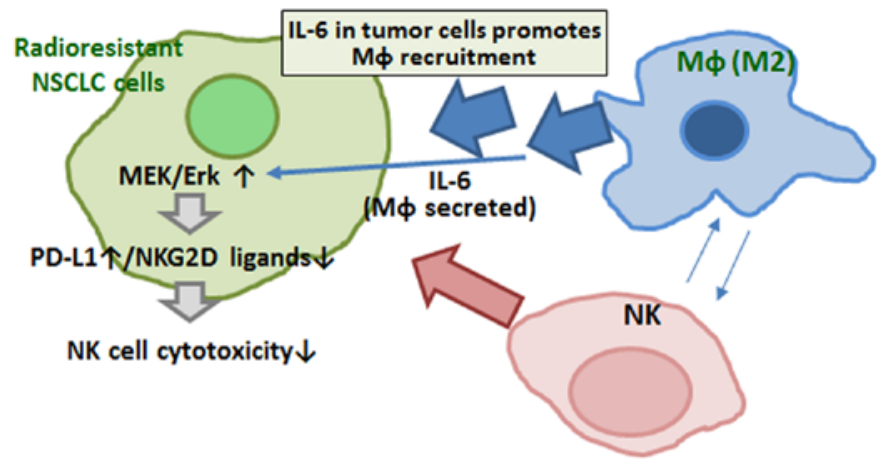

Figure 6. Examining the effects of MEK/Erk inhibition on natural killer (NK) cell cytotoxicity to radioresistant lung cancer cells treated with conditioned medium (CM) of PMA/IL-4-treated THP-1 cells. (A) NK cell cytotoxicity to radioresistant lung cancer cells after the addition of the MEK/Erk inhibitor, U0126, into the CM of PMA/IL-4-treated THP-1 cells. (B) NK cell cytotoxicity to radioresistant lung cancer cells after the addition of the MEK/Erk inhibitor or PD-L1 Ab into the CM of PMA/IL-4-treated THP-1 cells. (C) Schematic diagram summarizing the results of this study of the development of the additional resistance of radioresistant cells to NK cell cytotoxicity via increased macrophage infiltration. ${ }^{*} \mathrm{P}<0.05$ and ${ }^{* *} \mathrm{P}<0.01$.

tion $(13,31,32)$, and we also confirmed the importance of this interaction in modulating the NK cell action in our previous studies $(10,14,22)$. We found that THP-1 CM treatment also triggered the downregulation of NKG2D ligands (ULBP1, ULBP2, ULBP3, MICA and MICB) in radioresistant lung cancer cells (Fig. 3D), suggesting that this modulation may also be associated with the THP-1 CM effect.

IL-6 signaling was important in exerting the THP-1 CM effects. The immunosuppressive effects exerted by THP-1CM treatment may be due to the released cytokines by macrophages, such as IL-6 and IL-10 (33,34). We also detected high secreted IL-6 levels in the PMA/IL-4-treated THP-1 cells (Fig. 4A) (we observed a higher IL-6 secretion in PMA/IL-4-treated THP-1 cells than in PMA alone-treated cells). We then examined whether the presence of IL- 6 in THP-1 CM plays a critical role in exerting the THP-1CM effect in alteration of the PD-L1/NKG2D ligands in THP-1 CM-treated radioresistant lung cancer cells. IL-6 Ab was added to THP-1 CM when treating the radioresistant cells, and the PD-L1/NKG2D ligand levels in those cells were analyzed. We found that the THP-1 CM effects in altering the PD-L1/NKG2D ligand levels in radioresistant lung cancer cells were abolished upon the addition of IL-6 Ab; thus, decreased PD-L1 (Fig. 4B) and recovered NKG2D ligand levels (Fig. 4C) were observed. We then examined the effect of IL- $6 \mathrm{Ab}$ on the susceptibility of THP-1 CM-treated radioresistant cells to NK cell cytotoxicity. As shown in Fig. 4D, we observed an enhanced susceptibility of THP-1 CM-treated radioresistant lung cancer cells to NK cell cytotoxicity following the addition of IL- $6 \mathrm{Ab}$ into the co-culture. These data suggest an important role of IL-6 in triggering the THP-1 CM effect.

To confirm the role of IL-6 in influencing the susceptibility of radioresistant cells to NK cell cytotoxicity, hrIL- 6 was added to the mixture of tumor cells/NK cells. As shown in Fig. 4E, we observed an increased resistance of the radioresistant lung 
cancer cells to NK cell cytotoxicity, confirming the role of IL-6 in promoting the additional resistance of radioresistant lung cancer cells to NK cell cytotoxicity.

MEK/Erk signaling is the IL-6 downstream signaling pathway that is responsible for triggering the THP-1 CM effects. To reveal the signaling pathway that is important in triggering the THP-1 CM effect (in altering the PD-L1/NKG2D ligand levels), we added inhibitors of candidate signaling pathways to the THP-1 CM-treated radioresistant cells and examined which signaling pathway can abolish the THP-1 CM effect. For the candidate signaling pathways, we selected the pathways that have been previously reported to be involved in PD-L1 regulation in cancer cells, including JAK1/2 $(35,36)$, Stat3 $(37,38)$, Akt-mTOR (39), NF-kB (40), MAPK/Erk kinase (MEK), Erk $(41,42)$, PI3K/Akt $(39,43)$ and MAPK $(44)$. We first analyzed the PD-L1 level in the inhibitor-treated cells and observed the most significant reduction in PD-L1 level when the MEK/Erk inhibitor was added into THP-1 CM (Fig. 5A). These results were consistent with those of our previous study, demonstrating MEK/Erk signaling as the critical signaling pathway for mediating the PD-L1/NKG2D ligand level alterations in radioresistant lung cancer cells (10). The reduced PD-L1 protein level in THP-1 CM-treated radioresistant lung cancer cells upon treatment with the MEK/Erk inhibitor is shown in Fig. 5B.

We investigated whether the MEK/Erk signaling inhibition also affects the NKG2D ligand levels in THP-1 CM-treated radioresistant lung cancer cells. We found that inhibitor treatment recovered the decreased 5 NKG2D ligand levels (ULBP1, ULBP2, ULBP3, MICA and MICB) in the THP-1 CM-treated radioresistant lung cancer cells (Fig. 5C), indicating that the inhibition of the MEK/Erk signaling was effective not only in lowering the PD-L1 level, but also in recovering the NKG2D ligands levels in THP-1 CM-treated radioresistant cells.

To investigate whether the activation of MEK/Erk signaling in THP-1 CM-treated radioresistant cells was due to IL-6 signaling, we added IL- $6 \mathrm{Ab}$ and examined whether the activation of this signaling can be suppressed. As shown in Fig. 5D, we detected reduced p-Erk levels when IL-6 Ab was added to the THP-1 CM when treating radioresistant lung cancer cells.

Use of inhibitor of MEK/Erk signaling effectively blocked the THP-1 CM effect. We then examined whether the MEK/Erk signaling inhibition enhances the susceptibility of the THP-1 CM-treated radioresistant lung cancer cells to NK cell cytotoxicity. As shown in Fig. 6A (left panel, A549R26-1 data; right panel, H157R24-1 cells data), the addition of MEK/Erk signaling inhibitor enhanced the susceptibility of THP-1 CM-treated radioresistant lung cancer cells to NK cell cytotoxicity and the effect was similar to the effect shown by PD-L1 Ab (Fig. 6B), suggesting that the strategy of altering the PD-L1/NKG2D levels by these inhibitors may be an alternative way of recovering the THP-1 CM-induced resistance of radioresistant lung cancer cells to NK cell cytotoxicity. The schematic diagram shown in Fig. 6C summarizes our results.

\section{Discussion}

TAM infiltration to tumors in the tumor microenvironment is a widely accepted concept. Once this occurs, TAMs promote epithelial-mesenchymal transition (EMT) and cancer metastasis (1,45-47).

In this study, we observed higher TAM migration to radioresistant cells than to parental cells. In a previously published study (15), we demonstrated that IL-6-CCL2/CCL5 signaling triggers macrophage migration to tumor cells and that this was also important in enhancing macrophage migration to radioresistant cells than to parental cells.

TAMs are also known to play an immunosuppressive role to affect T-cell function. However, the role of TAMs in affecting NK cell-mediated antitumor cytotoxicity via affecting either tumor cells or NK cells has not yet been extensively investigated, at least to the best of our knowledge. Recently, the role of TAMs in affecting NK cell cytotoxicity through modulating the receptor level (NKG2D) on NK cells has been reported (8); however, to the best of our knowledge, no studies to date have focused on revealing the role of TAMs in influencing the ligand levels on tumor cells to affect their susceptibilities to NK cell cytotoxicity.

In a previous study, we demonstrated that radioresistant cells are more resistant to NK cell cytotoxicity than parental cells (10). In this study, we further found that higher numbers of macrophages infiltrated to radioresistant tumor cells, which in turn affected tumor cells to develop additional resistance of these cells to NK cell cytotoxicity. Thus, the emphasis of this study is on the development of additional resistance to NK cell cytotoxicity by increased macrophage infiltration. We suggest that such a malignant cycle influences tumors to become more aggressive.

In this study, we demonstrated that the development of additional resistance of radioresistant cells to NK cell cytotoxicity by increased macrophage migration was via altering the ligand levels (increasing PD-L1/decreasing NKG2D ligand level) on lung cancer cells that are important in tumor cell-NK cell interaction (29-32). However, Zhou et al (9) indicated that the NKG2D ligand levels on macrophages were upregulated upon co-culture with NK cells. As we have not examined the NKG2D level alteration on NK cells upon treatment with THP-1CM, further studies are warranted to reveal the THP-1 $\mathrm{CM}$ effect on alterations of NKG2D ligand levels (on tumor cells) and NKG2D receptor levels (on NK cells) in co-culture systems of tumor cells, THP-1 cells, and NK cells.

We proved the implication of IL-6 in exerting the THP-1 CM effect in decreasing NK cell cytotoxicity to lung cancer cells. IL-6 has been considered as an M1 macrophage marker (46). However, IL- 6 secretion by M2 macrophages has also been reported $(33,34)$, and high levels of IL-6 were detected in TAMs isolated from glioblastoma patients (48). We also detected high IL-6 levels in PMA/IL-4 treated THP-1 cells. Based on our data, we suggest that the IL- 6 secreted by THP- 1 cells may be the one of the major cytokines that trigger the MEK/Erk activation in radioresistant lung cancer cells. Jeong et al (49) demonstrated the M2 TAM-activated MEK/Erk signaling pathway in breast cancer cells, and IL-6 triggered-MEK/ERK activation in other types of cancer cells has also previously been demonstrated $(50,51)$. However, IL-6 may not be the only molecule contained in THP-1 CM and may trigger MEK/Erk activation. The possible implication of other cytokines, including IL-10, which is shown to exist at the highest level in M2 type macrophages (52) in inducing the resistance of tumor cells to NK cell cytotoxicity, warrants further investigation in the future. 
These studies emphasize the detrimental effects of macrophage-secreted IL-6 on triggering immunosuppressive effects on NK cell cytotoxicity. However, using anti-IL-6 agents may result in complex and unforeseen outcomes considering the complexity of the physiological activities of IL-6 in producing both pro- and anti-inflammatory effects in the immune system (53). Therefore, we hypothesized that targeting the MEK/Erk signaling pathway may be a better therapeutic option than using anti-IL-6 agents. Indeed, we observed similar effects of using MEK/Erk inhibitor in increasing the susceptibility of THP-1 CM-treated radioresistant lung cancer cells than using each agent individually (Fig. 6B). The implication of MEK/Erk signaling in developing the resistance to NK cell cytotoxicity (via altering PD-L1/NKG2D ligand levels) is consistent with the results we have previously demonstrated (10). However, while previous studies were focused on the mechanisms through which radioresistant cells develop an enhanced resistance to NK cell cytotoxicity than parental cells, this study emphasizes the importance of this signaling in mediating the effects of macrophages after infiltration to radioresistant tumors. Therefore, we hypothesize that the strategy of targeting this signaling strategy may be specifically effective in treating lung cancer patients at the radioresistant stage.

Clinical trials targeting PD-L1/PD-1 axis in treating lung cancer so far have not yielded satisfactory results (54). In addition, no specific targeted therapies on lung cancer at the radioresistant stage are currently available. The use of the $\mathrm{MEK} /$ Erk pathway inhibitor may prove to be an alternative therapeutic option than using the PD-L1 Ab-using immunotherapy for the treatment of radioresistant lung cancer.

\section{Acknowledgements}

The authors would like to thank Mrs. Laura Finger of the Department of Radiation Oncology at the University of Rochester Medical Center for providing assistance with the manuscript preparation.

\section{Funding}

Richard Bell Endowed Professorship by University of Rochester Medical Center. Endowed professorship is the endowed fund to support the academic mission of the PI of the laboratory YuC.

\section{Availability of data and materials}

All data generated or analyzed during this study are included in this published article.

\section{Authors' contributions}

MS and YoC performed the NK cell cytotoxicity tests, migration tests, qPCR analyses and animal experiments. LX isolated the NK cells and maintained primary NK cell culture and performed IHC staining. RZ and XX performed IF staining and western blot analyses. YT performed IL-6 ELISA. PCK and $\mathrm{YuC}$ analyzed and interpreted the data. SOL was a major contributor in designing the experiments and the writing of the manuscript. All authors have read and approved the final manuscript.

\section{Ethics approval and consent to participate}

All animal experiments were performed under the supervision and guidelines of the University of Rochester Medical Center Animal Care and Use Committee. The facilities and programs for animal care at the University of Rochester comply with state and federal laws and NIH policies. The entire Animal Resource program is accredited by the Association for Assessment and Accreditation of Laboratory Animal Care International (AAALAC).

\section{Consent for publication}

Not applicable.

\section{Competing interests}

The authors declare that they have no competing interests.

\section{References}

1. Qian BZ and Pollard JW: Macrophage diversity enhances tumor progression and metastasis. Cell 141: 39-51, 2010.

2. Mantovani A and Sica A: Macrophages, innate immunity and cancer: Balance, tolerance, and diversity. Curr Opin Immunol 22: 231-237, 2010.

3. Arango Duque G and Descoteaux A: Macrophage cytokines: Involvement in immunity and infectious diseases. Front Immunol 5: 491, 2014.

4. Ojalvo LS, King W, Cox D and Pollard JW: High-density gene expression analysis of tumor-associated macrophages from mouse mammary tumors. Am J Pathol 174: 1048-1064, 2009.

5. Kuang DM, Zhao Q, Peng C, Xu J, Zhang JP, Wu C and Zheng L: Activated monocytes in peritumoral stroma of hepatocellular carcinoma foster immune privilege and disease progression through PD-L1. J Exp Med 206: 1327-1337, 2009.

6. Williams CB, Yeh ES and Soloff AC: Tumor-associated macrophages: Unwitting accomplices in breast cancer malignancy. NPJ Breast Cancer 2: 2, 2016.

7. Krneta T, Gillgrass A, Poznanski S, Chew M, Lee AJ, Kolb M and Ashkar AA: M2-polarized and tumor-associated macrophages alter NK cell phenotype and function in a contact-dependent manner. J Leukoc Biol 101: 285-295, 2017.

8. Crane CA, Austgen K, Haberthur K, Hofmann C, Moyes KW, Avanesyan L, Fong L, Campbell MJ, Cooper S, Oakes SA, et al: Immune evasion mediated by tumor-derived lactate dehydrogenase induction of NKG2D ligands on myeloid cells in glioblastoma patients. Proc Natl Acad Sci USA 111: 12823-12828, 2014.

9. Zhou Z, Zhang C, Zhang J and Tian Z: Macrophages help NK cells to attack tumor cells by stimulatory NKG2D ligand but protect themselves from NK killing by inhibitory ligand Qa-1. PLoS One 7: e36928, 2012.

10. Shen MJ, Xu LJ, Yang L, Tsai Y, Keng PC, Chen Y, Lee SO and Chen Y: Radiation alters PD-L1/NKG2D ligand levels in lung cancer cells and leads to immune escape from NK cell cytotoxicity via IL-6-MEK/Erk signaling pathway. Oncotarget 8: 80506-80520, 2017.

11. Mu CY,Huang JA, Chen Y, Chen C and Zhang XG: High expression of PD-L1 in lung cancer may contribute to poor prognosis and tumor cells immune escape through suppressing tumor infiltrating dendritic cells maturation. Med Oncol 28: 682-688, 2011.

12. Afreen S and Dermime S: The immunoinhibitory B7-H1 molecule as a potential target in cancer: Killing many birds with one stone. Hematol Oncol Stem Cell Ther 7: 1-17, 2014.

13. Nausch $\mathrm{N}$ and Cerwenka A: NKG2D ligands in tumor immunity. Oncogene 27: 5944-5958, 2008.

14. Yang L, Shen M, Xu LJ, Yang X, Tsai Y, Keng PC, Chen Y and Lee SO: Enhancing NK cell-mediated cytotoxicity to cisplatin-resistant lung cancer cells via MEK/Erk signaling inhibition. Sci Rep 7: 7958, 2017.

15. Wang X, Yang X, Tsai Y, Yang L, Chuang KH, Keng PC, Lee SO and Chen Y: IL-6 mediates macrophage infiltration after irradiation via up-regulation of CCL2/CCL5 in non-small cell lung cancer. Radiat Res 187: 50-59, 2017. 
16. Aarons CB, Bajenova O, Andrews C, Heydrick S Bushell KN, Reed KL, Thomas P, Becker JM and Stucchi AF: Carcinoembryonic antigen-stimulated THP-1 macrophages activate endothelial cells and increase cell-cell adhesion of colorectal cancer cells. Clin Exp Metastasis 24: 201-209, 2007.

17. Genin M, Clement F, Fattaccioli A, Raes M and Michiels C: M1 and M2 macrophages derived from THP-1 cells differentially modulate the response of cancer cells to etoposide. BMC Cancer 15: 577, 2015.

18. Korzeniewski C and Callewaert DM: An enzyme-release assay for natural cytotoxicity. J Immunol Methods 64: 313-320, 1983.

19. Decker T and Lohmann-Matthes ML: A quick and simple method for the quantitation of lactate dehydrogenase release in measurements of cellular cytotoxicity and tumor necrosis factor (TNF) activity. J Immunol Methods 115: 61-69, 1988.

20. Shi L, Lin H, Li G, Sun Y, Shen J, Xu J, Lin C, Yeh S, Cai X and Chang C: Cisplatin enhances NK cells immunotherapy efficacy to suppress $\mathrm{HCC}$ progression via altering the androgen receptor (AR)-ULBP2 signals. Cancer Lett 373: 45-56, 2016.

21. Smith SM, Wunder MB, Norris DA and Shellman YG: A simple protocol for using a LDH-based cytotoxicity assay to assess the effects of death and growth inhibition at the same time. PLoS One 6: e26908, 2011.

22. Xu L, Chen X, Shen M, Yang DR, Fang L, Weng G, Tsai Y, Keng PC, Chen Y and Lee SO: Inhibition of IL-6-JAK/Stat3 signaling in castration-resistant prostate cancer cells enhances the NK cell-mediated cytotoxicity via alteration of PD-L1/NKG2D ligand levels. Mol Oncol 12: 269-286, 2018.

23. Lee Y, Auh SL, Wang Y, Burnette B, Wang Y, Meng Y, Beckett M, Sharma R, Chin R, Tu T, et al: Therapeutic effects of ablative radiation on local tumor require $\mathrm{CD}^{+} \mathrm{T}$ cells: Changing strategies for cancer treatment. Blood 114: 589-595, 2009.

24. Livak KJ and Schmittgen TD: Analysis of relative gene expression data using real-time quantitative PCR and the 2(-Delta Delta C(T)) method. Methods 25: 402-408, 2001.

25. Xu H, Lai W, Zhang Y, Liu L, Luo X, Zeng Y, Wu H, Lan Q and Chu Z: Tumor-associated macrophage-derived IL-6 and IL-8 enhance invasive activity of LoVo cells induced by PRL-3 in a KCNN4 channel-dependent manner. BMC Cancer 14: 330, 2014.

26. Zhou Y, Yoshida S, Kubo Y, Yoshimura T, Kobayashi Y, Nakama T, Yamaguchi M, Ishikawa K, Oshima Y and Ishibashi T: Different distributions of M1 and M2 macrophages in a mouse model of laser-induced choroidal neovascularization. Mol Med Rep 15: 3949-3956, 2017.

27. Nawaz A, Aminuddin A, Kado T, Takikawa A, Yamamoto S, Tsuneyama K, Igarashi Y, Ikutani M, Nishida Y, Nagai Y, et al: CD206 ${ }^{+}$M2-like macrophages regulate systemic glucose metabolism by inhibiting proliferation of adipocyte progenitors. Nat Commun 8: 286, 2017.

28. Xu L, Shen M, Chen X, Yang DR, Tsai Y, Keng PC, Lee SO and Chen Y: In vitro-induced M2 type macrophages induces the resistance of prostate cancer cells to cytotoxic action of NK cells. Exp Cell Res 364: 113-123, 2018.

29. Benson DM Jr, Bakan CE, Mishra A, Hofmeister CC, Efebera Y, Becknell B, Baiocchi RA, Zhang J, Yu J, Smith MK, et al: The PD-1/PD-L1 axis modulates the natural killer cell versus multiple myeloma effect: A therapeutic target for CT-011, a novel monoclonal anti-PD-1 antibody. Blood 116: 2286-2294, 2010.

30. Huang BY, Zhan YP, Zong WJ, Yu CJ, Li JF, Qu YM and Han S: The PD-1/B7-H1 pathway modulates the natural killer cells versus mouse glioma stem cells. PLoS One 10: e0134715, 2015.

31. Bae DS, Hwang YK and Lee JK: Importance of NKG2D-NKG2D ligands interaction for cytolytic activity of natural killer cell. Cell Immunol 276: 122-127, 2012

32. González S, López-Soto A, Suarez-Alvarez B, López-Vázquez A and López-Larrea C: NKG2D ligands: Key targets of the immune response. Trends Immunol 29: 397-403, 2008.

33. Niemand C, Nimmesgern A, Haan S, Fischer P, Schaper F, Rossaint R, Heinrich PC and Müller-Newen G: Activation of STAT3 by IL-6 and IL-10 in primary human macrophages is differentially modulated by suppressor of cytokine signaling 3 . J Immunol 170: 3263-3272, 2003.

34. Li YY, Hsieh LL, Tang RP, Liao SK and Yeh KY: Interleukin-6 (IL-6) released by macrophages induces IL-6 secretion in the human colon cancer HT-29 cell line. Hum Immunol 70: 151-158, 2009.

35. Bellucci R, Martin A, Bommarito D, Wang K, Hansen SH, Freeman GJ and Ritz J: Interferon- $\gamma$-induced activation of JAK1 and JAK2 suppresses tumor cell susceptibility to NK cells through upregulation of PD-L1 expression. OncoImmunology 4: e1008824, 2015 .
36. Ikeda S, Okamoto T, Okano S, Umemoto Y, Tagawa $\mathrm{T}$, Morodomi Y, Kohno M, Shimamatsu S, Kitahara H, Suzuki Y, et al: PD-L1 is upregulated by simultaneous amplification of the PD-L1 and JAK2 genes in non-small cell lung cancer. J Thorac Oncol 11: 62-71, 2016

37. Fujita Y, Yagishita S, Hagiwara K, Yoshioka Y, Kosaka N, Takeshita F, Fujiwara T, Tsuta K, Nokihara H, Tamura T, et al: The clinical relevance of the miR-197/CKS1B/STAT3-mediated PD-L1 network in chemoresistant non-small-cell lung cancer. Mol Ther 23: 717-727, 2015.

38. Marzec M, Zhang Q, Goradia A, Raghunath PN, Liu X, Paessler M, Wang HY, Wysocka M, Cheng M, Ruggeri BA, et al: Oncogenic kinase NPM/ALK induces through STAT3 expression of immunosuppressive protein CD274 (PD-L1, B7-H1). Proc Natl Acad Sci USA 105: 20852-20857, 2008.

39. Lastwika KJ, Wilson W III, Li QK, Norris J, Xu H, Ghazarian SR, Kitagawa H, Kawabata S, Taube JM, Yao S, et al: Control of PD-L1 expression by oncogenic activation of the AKT-mTOR pathway in non-small cell lung cancer. Cancer Res 76: 227-238, 2016.

40. Gowrishankar K, Gunatilake D, Gallagher SJ, Tiffen J, Rizos H and Hersey P: Inducible but not constitutive expression of PD-L1 in human melanoma cells is dependent on activation of NF- $\kappa B$. PLoS One 10: e0123410, 2015.

41. Yamamoto R, Nishikori M, Tashima M, Sakai T, Ichinohe T, Takaori-Kondo A, Ohmori K and Uchiyama T: B7-H1 expression is regulated by MEK/ERK signaling pathway in anaplastic large cell lymphoma and Hodgkin lymphoma. Cancer Sci 100: 2093-2100, 2009.

42. Chen N1, Fang W, Zhan J, Hong S, Tang Y, Kang S, Zhang Y, He X, Zhou T, Qin T, et al: Upregulation of PD-L1 by EGFR activation mediates the immune escape in EGFR-driven NSCLC: Implication for optional immune targeted therapy for NSCLC patients with EGFR mutation. J Thorac Oncol 10: 910-923, 2015.

43. Xu C, Fillmore CM, Koyama S, Wu H, Zhao Y, Chen Z, Herter-Sprie GS, Akbay EA, Tchaicha JH, Altabef A, et al: Loss of Lkb1 and Pten leads to lung squamous cell carcinoma with elevated PD-L1 expression. Cancer Cell 25: 590-604, 2014.

44. Noh H,Hu J, Wang X, Xia X, Satelli A and Li S: Immune checkpoint regulator PD-L1 expression on tumor cells by contacting CD11b positive bone marrow derived stromal cells. Cell Commun Signal 13: 14, 2015.

45. Dehai C, Bo P, Qiang T, Lihua S, Fang L, Shi J, Jingyan C, Yan Y, Guangbin W and Zhenjun Y: Enhanced invasion of lung adenocarcinoma cells after co-culture with THP-1-derived macrophages via the induction of EMT by IL-6. Immunol Lett 160: 1-10, 2014.

46. Condeelis J and Pollard JW: Macrophages: Obligate partners for tumor cell migration, invasion, and metastasis. Cell 124: 263-266, 2006

47. Liu CY, Xu JY, Shi XY, Huang W, Ruan TY, Xie P and Ding JL: M2-polarized tumor-associated macrophages promoted epithelialmesenchymal transition in pancreatic cancer cells, partially through TLR4/IL-10 signaling pathway. Lab Invest 93: 844-854, 2013.

48. Hattermann K, Sebens S, Helm O, Schmitt AD, Mentlein R, Mehdorn HM and Held-Feindt J: Chemokine expression profile of freshly isolated human glioblastoma-associated macrophages/ microglia. Oncol Rep 32: 270-276, 2014.

49. Jeong SK, Kim JS, Lee CG, Park YS, Kim SD, Yoon SO, Han DH, Lee KY, Jeong MH and Jo WS: Tumor associated macrophages provide the survival resistance of tumor cells to hypoxic microenvironmental condition through IL-6 receptor-mediated signals. Immunobiology 222: 55-65, 2017.

50. Lee SO, Lou W, Johnson CS, Trump DL and Gao AC: Interleukin-6 protects $\mathrm{LNCaP}$ cells from apoptosis induced by androgen deprivation through the Stat3 pathway. Prostate 60: 178-186, 2004.

51. Yu H, Pardoll D and Jove R: STATs in cancer inflammation and immunity: A leading role for STAT3. Nat Rev Cancer 9: 798-809, 2009.

52. Jeannin P, Duluc D and Delneste Y: IL-6 and leukemia-inhibitory factor are involved in the generation of tumor-associated macrophage: Regulation by IFN- $\gamma$. Immunotherapy 3 (Suppl 4): 23-26, 2011.

53. Scheller J, Chalaris A, Schmidt-Arras D and Rose-John S: The pro- and anti-inflammatory properties of the cytokine interleukin-6. Biochim Biophys Acta 1813: 878-888, 2011.

54. Topalian SL, Hodi FS, Brahmer JR, Gettinger SN, Smith DC, McDermott DF, Powderly JD, Carvajal RD, Sosman JA, Atkins MB, et al: Safety, activity, and immune correlates of anti-PD-1 antibody in cancer. N Engl J Med 366: 2443-2454, 2012. 